\title{
Future Broadband Access Network Challenges
}

\author{
S. R. Abdollahi, H.S. Al-Raweshidy, R. Nilavalan, and Abolghasem Darzi* \\ WNCC Group, School of Eng. and Design, Brunel University, Uxbridge, Middlesex, UB8 3PH, UK. \\ E-mail: \{seyedreza.abdollahi, hamed al-raweshidy, and rajagopal.nilavalan \}@brunel.ac.uk \\ * Telecommunication Group, Sare Engineering Inc., I. R. of Iran, E-mail: ghasem.darzi@sare-eng.com
}

\begin{abstract}
The optical and wireless communication systems convergence will activate the potential capacity of photonic technology for providing the expected growth in interactive video, voice communication and data traffic services that are cost effective and a green communication service. The last decade growth of the broadband internet projects the number of active users will grow to over 2 billion globally by the end of 2014. Enabling the abandoned capacity of photonic signal processing is the promising solution for seamless transportation of the future consumer traffic demand. In this paper, the future traffic growth of the internet, wireless worldwide subscribers, and the end-users during the last and next decades is investigated. The challenges of the traditional access networks and Radio over Fiber solution are presented.
\end{abstract}

\section{INTRODUCTION}

The rapidly changing face of data communications and telecommunications has seen a continued growth in the need to transfer enormous amounts of information across large distances. The technologies that were used extensively in the past, such as coaxial cable, satellite and microwave radio for transferring information were very quickly running out of the capacity.

There was a growing requirement to provide a communication medium suitable to noisy industrial environment where there is a need for data communications. This was addressed with the introduction of fiber optic communication systems. Fiber optic transmission has become one of the most exciting and rapidly changing fields in telecommunication engineering. Fiber optic communication systems have many advantages over more conventional transmission systems. They are less affected by noise, are completely unaffected by electromagnetic interference (EMI) and radio frequency interference (RFI), do not conduct electricity and therefore, provide electrical isolation, are completely unaffected by lightning and high voltage switching, and carry extremely high data transmission rates over very long distances, [1], [2]. Growing the future network traffic about hundred Exabyte in video, data, mobile and wireless communication has been already been presented, [3]. Transportation of the demanded huge traffic requires enormous network transmission bandwidth than the present infrastructure capacity. By activating the vast capacity of the optical communication network and system and further advances in technology, this scheme will become available in the near future. As a result, research activity in the field of optical networks and radio over fiber has been grown very fast and steady over the last several years. This is because the optical communication is a promising choice to fulfill the ever-increasing demand on bandwidth via the vast available capacity of the fiber optics and its economic cost. In another view, wireless communication is entering a new phase where the focus is shifting from voice to multimedia services. Present consumers are no longer interested in the underlying technology; they simply need reliable and cost effective communication systems that can support anytime, anywhere, any media that they want, [1].

The paper is organized as follows. In Section II, the last decade growth in subscribing the internet and wireless network worldwide in diverse domain of services such as wireless and fixed broadband, landline and mobile communication are discussed. In Section III, we have discussed the solutions for transportation with huge traffic demand, according with the expected growth in interactive video, voice communication and data traffic for providing the cost effective communication services. In Section IV, the Radio over Fiber technique and system as a vast capacity infrastructure and superstructure of the promising solution for supporting super-broadband services of the future enduser as a reliable, cost-effective and environment friendship technology and sample simulation results are presented. Finally, concluding remarks are given in Section V.

\section{GROWTH OF INTERNET AND WIRELESS NETWORK}

Broadband wireless sits at the confluence of two of the most remarkable growth stories of the telecommunication industry in recent years.

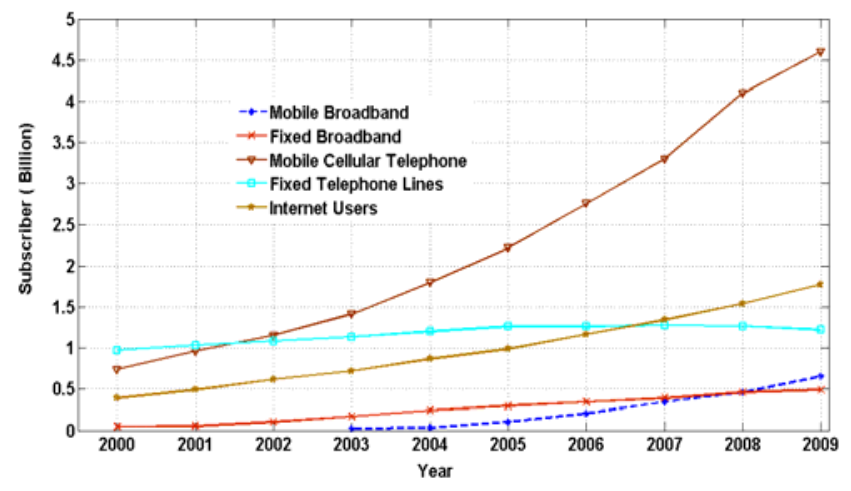

Fig. 1. Worldwide subscriber growth for mobile telephony, internet usage and broadband access users.

Both wireless and broadband have their own enjoyed rapid mass-market adoption. Wireless mobile services grow 
from 11 million subscribers worldwide in 1990 to more than 4.6 billion by the end of 2009. In fact, the world largest manufacture of the mobile phone has forecasted that the world will have more than 3 billion mobile users by the 2010. In addition, the number of active users accessing the internet via mobile broadband will grow to over 2 billion globally by the end 2014. Furthermore, the fixed broadband subscribers amounted to only 57,000 in 1998 were jumped to 487 million subscribers by the end of 2009. It is projected that this number will exceed 680 million in another four years by 2013, despite the current economic situation [2]. The growth of the telephone subscribers, broadband and Internet users during the last decade has been depicted in Fig. 1.

The availability of a wireless solution for broadband could potentially accelerate this growth. There are major questions to answer to justify such growth including: (1) what are the applications that derive this growth? Broadband users worldwide are finding that it dramatically changes how we share information, conduct business and seek entertainment, (2) Will combining the convenience of wireless with the rich performance of broadband be the next frontier for the growth in the industry?, (3) Can such a combination be technically and commercially viable?, and (4) Can wireless deliver broadband application and services that are of interest to the end-user? Many industry observers believe so.

\section{TRANSPORTATION OF THE FUTURE TRAFFIC}

The high speed broadband penetration and ongoing growth of the internet traffic away from residential and business customers' home have been placing a huge bandwidth demand on the underlying telecommunication infrastructure. There will be three waves of internet video. The first phase is experiencing a growth of internet video as viewed on the PC, the second phase will see a rise in internet delivery of video to the TV, and the third phase will involve a surge in video communications. Each phase will impact a different aspect of the network. The first two phases will be felt primarily in the metro and access networks, while the third will impact the core. In addition to internet video, there is very high growth in the IP transport of cable and IPTV video on-demand services, [2]. Only the virtually unlimited bandwidth of a fiber-optic access network can provide such capability both for wired and wireless connectivity.

As shown in Fig. 2 (a) and (b), globally, mobile data traffic will double every year, and reach over 2 exabytes per month by 2013. Furthermore, mobile data traffic will grow from 1 petabyte per month to 1 exabyte per month in half the time it took fixed data traffic to do so. Mobile voice service is already considered a necessity by many, and mobile data, video, and TV are now becoming an essential part of the end-users' lives. According to Fig. 1, the number of subscribers is growing rapidly and bandwidth demand due to data/video is increasing. There is a need for backhaul capacity to increase for mobile broadband, data access, and video services to engage the end-users as well as keep cost in check.



(a)

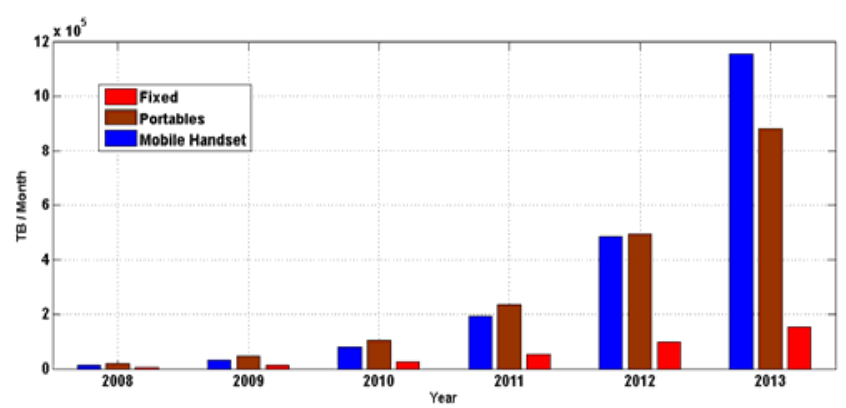

(b)

Fig. 2. The traffic growth of (a): the services type of traffic, (b): the wireless traffic.

In order to deploy next-generation mobile networks, there is a greater need for service portability and interoperability with the proliferation of mobile and portable digital devices, there is an imminent need for network to allow for all these devices to be connected seamlessly.. The expansion of wireless ubiquity will drive an increased volume of consumers to access and rely on mobile networks creating a need for greater economies of scale and lower perbit cost. In response to this remarkable development, the metro and core networks of the telecommunication infrastructure have experienced a tremendous growth in bandwidth and capacity with the wide deployment of fiberoptic technology in the past decade [4].

Data traffic speeds in metro and long-haul system are evolving from 10 Gbps to 40 Gbps transmission, 100 Gbps per wavelength channel system is taking shape as next step for core and metro networks [5]. Access bandwidth requirements for delivering multi-channel high-definition television (HDTV) signals and online gaming services are expected to grow to gigabits per second in the near future. However, the current subscriber access networks have not been scaled up commensurately. To avoid being the bottleneck in the last miles and last meters, and exploit the benefits of both wired and wireless technologies, carriers and service provides are actively seeking a convergent network architecture to deliver multiple services to serve both fixed and mobile users. In this regard, optical-wireless access 
technologies have been considered the most promising solution to increase the capacity, coverage, bandwidth, and mobility in environments such as conference centers, airports, hotels, shopping malls, and ultimately to homes and small offices.

Therefore, the demand for use of the radio spectrum is very high, with terrestrial mobile phone and broadband internet systems being just one of many applications vying for suitable bandwidth. These applications require the systems to operate reliably in non-line-of-sight environments with a propagation distance of $0.5-30 \mathrm{~km}$, and at velocities up to $100 \mathrm{~km} / \mathrm{h}$ or higher. This operating environment limits the maximum RF frequency, as operating at very high frequency near mm-wave results in excessive channel path loss, and excessive Doppler spread at high velocity. This limits the spectrum available for mobile application, making the value of the radio spectrum extremely high.

\section{A. Optical Switching Paradigm}

The main challenges of the core and metro networks for the future traffic transportation are the present active electrical switching and routing systems, because of insertion delay for procession the packet for switching in electrical domain. So, it needs time for conversion of the signal from optical to electrical domain and vice versa, in addition the synchronization and data retiming processing time. In particular, a great part of research in optical networks is dedicated to transparency in optical networks in order to bypass the Optical/Electrical/Optical $(\mathrm{O} / \mathrm{E} / \mathrm{O})$ conversions in the intermediate nodes of the network. Thus, a number of network protocols (such as MPLS, GMPLS, etc.) together with switching strategies (circuit- burst- or packet-switching) are proposed for data transparency in the network. Among the switching strategies, the burst switching is more compatible with the current optoelectronic technologies in terms of data transparency and switching speed. The packet switching is more efficient for data communication, but due to the limited speed on electronics (in comparison with the present optical bandwidth in fiber optics) and the insufficient evolution of all-optical signal processing alternatives, the packet based optical networks are not still a practical solution in transparent optical networks, Fig. 3.

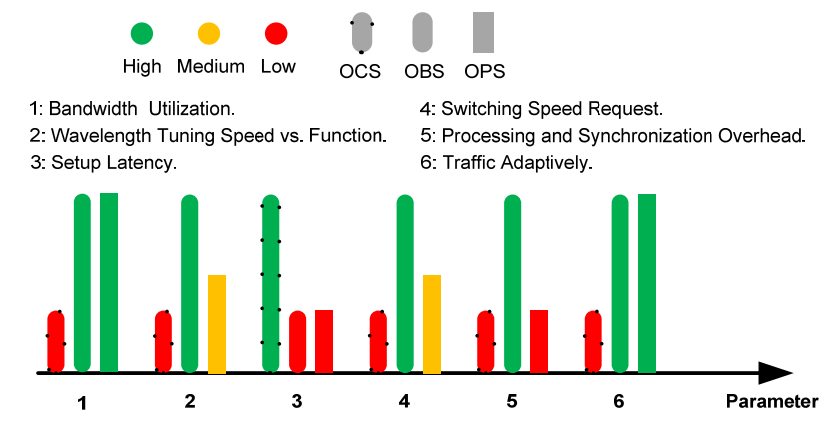

Fig. 3. The comparison between all-optical switching technologies.

Optical packet switching (OPS) became a viable candidate because it is a purely-connectionless networking solution that is fully compatible with IP-centric data traffic and offers the finest network granularity, optimum bandwidth utilization, flexibility, high-speed, and its ability to use the resources economically.

An OPS implies higher requisites than OBS because of packet processing on the fly. A more feasible approach towards the implementation of OPS considers the use of synchronously transmitted packets with fixed lengths. However, in this case the hardware overhead is on the implementation of the packet synchronizer at the input. Despite their feasibility limitations, OPS demonstrators assisted the development of numerous ultra-fast switching and processing techniques regarding wavelength conversion, header encoding/decoding and processing, label swapping, fast clock extraction, and regeneration.

The main challenges in OPS are the implementation of the optical header processing mechanism, the development of an intelligent switch controller, the realization of ultra fast switching at a nanosecond timescale and the exploitation on buffering mechanisms to reduce packet blocking [6-9].

\section{B. Wireless and Wired Access Network}

Currently, wired and wireless services are separately provided by two independent physical networks, wired networks based on Fiber to the home (FTTH) access technologies provide huge bandwidth to users, but are not flexible enough to allow roaming connections. On the other hand, wireless networks offer mobility to users, but do not possess abundant bandwidth to meet the ultimate demand for multi-channel video services with high definition quality. Therefore, seamless integration of wired and wireless services for future-proof access networks will lead to the convergence of ultimate high bandwidth for both fixed and mobile users in a single, low-cost transport platform, This can be accomplished by using the developed hybrid optical and wireless networks, which not only can transmit wireless signals over fiber at the BS, but also simultaneously provide the wired services to the end users.

Broadband access not only provides faster web surfing and quicker file download, but also enables several multimedia applications such as real-time audio and video streaming, multimedia conferencing, and the interactive gaming. Broadband connections are also being used for voice telephony using Voice-over-Internet-Protocol (VoIP) technology. More advanced broadband access systems, such as FTTH and Very high data rate DSL (VDSL), enable such applications as entertainment-quality video, including HDTV and Video on Demand (VoD). As the broadband market continues to grow, several new applications are likely to emerge and it is difficult to predict which one will succeed in the future.

So, what is the broadband wireless? Broadband wireless is about bringing the broadband experience to a wireless context, which offers users certain unique benefits and convenience. There are two fundamentally different types of broadband wireless services. The first attempts to provide a set of services similar to that of the traditional fixed-line broadband but using wireless as the medium of transmission. 
This type, called fixed wireless broadband, can be thought of as a competitive alternative to DSL or cable modem. The second type of broadband wireless, called mobile broadband, offers the additional functionality of probability, nomadicity, and mobility. Mobile broadband attempts to bring broadband applications to new user experience scenarios and hence can offer the end-user a very different value proposition.

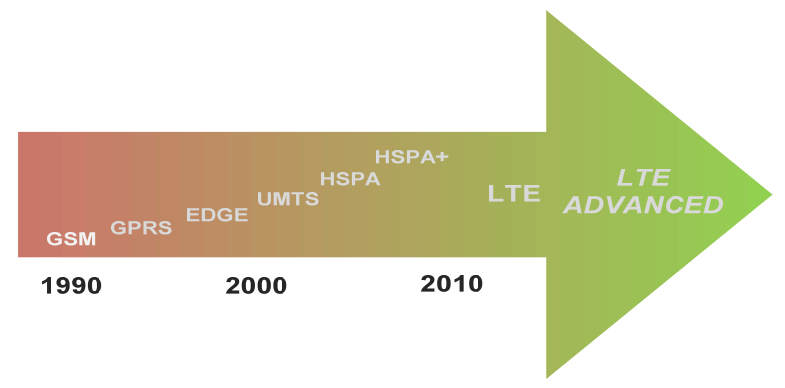

Fig. 4. Evolution of the GSM family toward Green Communication Technology.

For example, WiMax (worldwide interoperability for microwave access) technology and Long Term Evolution (LTE) there is a new radio platform technology that will allow operators to achieve even higher peak throughputs than High Speed Packet Access evolution) HSPA+) in higher spectrum bandwidth. The overall objective for LTE is providing an extremely high performance radio-access technology that offers full vehicular mobility and that readily coexist with HSPA and earlier networks. Because of scalable bandwidth, operators will be able to easily migrate their networks and users from HSPA to LTE over time. LTE assumes a full IP network architecture. Fig. 4 shows the evolution of GSM family toward the advanced LTE [13][14]. LTE uses OFDMA (Orthogonal Frequency Division Multiplexing Access) on the downlink, which is well suited to achieve high peak data rates in high spectrum bandwidth. Achieving peak rates in the $100 \mathrm{Mbps}$ range with wider radio channels, however, would result in highly complex terminals and is not practical with the current technology.

\section{THE RADIO OVER FIBER SOLUTION}

Radio-over-Fiber as a solution has been addressing how to distribute broadband wireless signals in access networks, including dynamic allocation of resources. Radio-over-Fiber system (RoF) is the technique of modulating the radio frequency (RF) sub-carrier onto an optical carrier for distribution over a fiber network. RoF technique has been considered a cost-effective and reliable solution for the distribution of the future wireless access networks by using optical fiber with vast transmission bandwidth capacity. RoF link is used in remote antenna applications to distribute signals for Microcell or Picocell Base Station. As shown in Fig. 8, in the remote antenna application, the downlink RF signals are distributed from a central station (CS) to many base station (BS) known as Radio Access Point (RAP) through the fibers. The uplink signals received at RAP are sent back to the CS for any signal processing. RoF has the following main features: (1) it is transparent to bandwidth or modulation techniques. (2) Simple and small BSs. (3) Centralized operation is possible.

New wireless subscribers are signing up at an increasing demand of more capacity for ultra-high data rate transfer at speeds of $1 \mathrm{Gbp} / \mathrm{s}$ and up regarding, while the radio spectrum is limited. This requirement of more bandwidth allocation, places heavy burden on the current operating radio spectrum and causes spectral congestion at lower microwave frequency. Millimeter Wave (mm-Wave) communication system offers a unique way to resolve these problems. Furthermore, to reduce the total cost and serve as many users as possible, $60 \mathrm{GHz}$ systems show excellent advantages for the future super-broadband data services delivering in terms of data rate and power consumption. Therefore, developing a cost effective means of generating optical millimeter-wave signals at frequencies above $60 \mathrm{GHz}$ is of great interest [15].

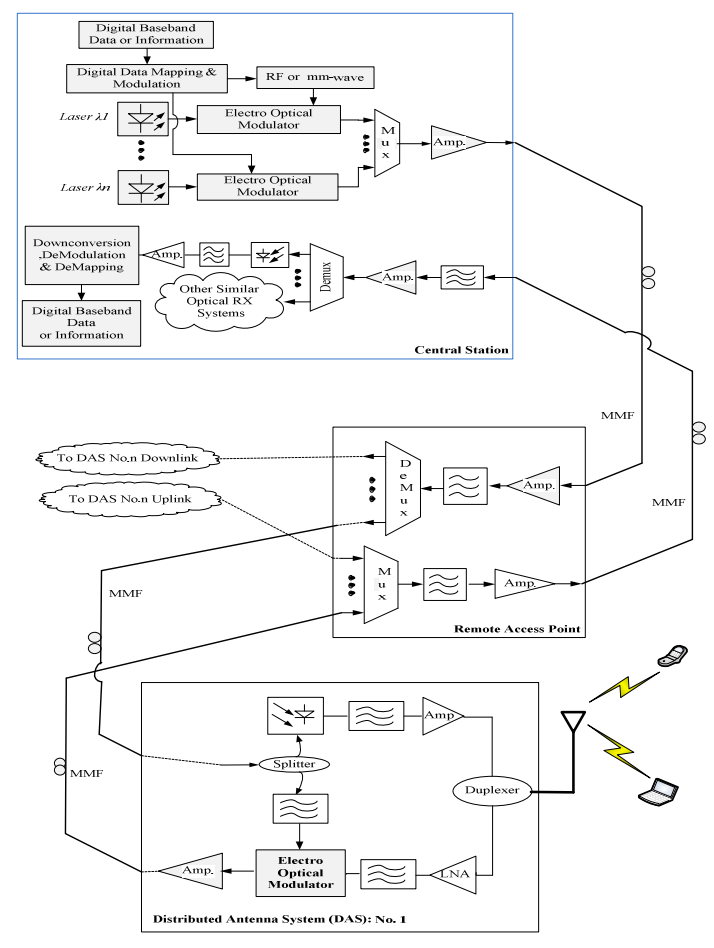

Fig. 5. Block diagram of: CS, RAP and distributed antenna system of designed indoor RoF system.

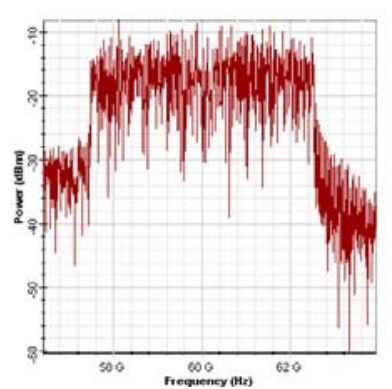

(a)

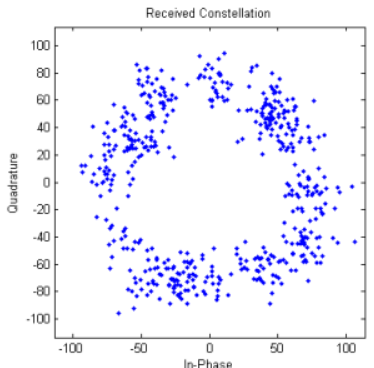

(b) 


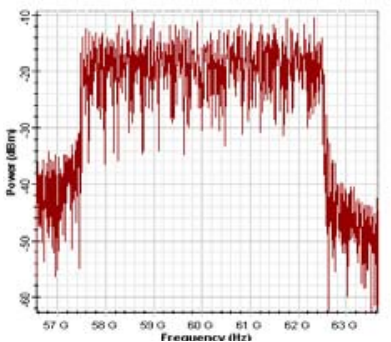

(c)

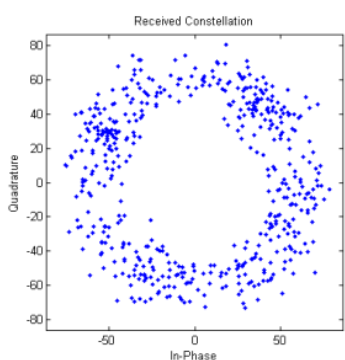

(d)
Fig. 6. Downlink received signal, (a) Spectrum, (b) Constellation, and Uplink received signal, (c) Spectrum, (d) Constellation, over 1.5 Km MMF

The details of a sample Radio over Multi-Mode Fiber (MMF) system block diagrams for indoor application are shown in Fig. 5. In this system simulation that has been performed by using Optiwave-Optisystem and Matlab environments, the attenuation and dispersion of the MMF are assumed $2 \mathrm{~dB} / \mathrm{Km}$ and $100 \mathrm{ps} / \mathrm{nm} / \mathrm{km}$, respectively, and a 10 $\mathrm{mW}$ laser source at $1550 \mathrm{~nm}$ is used at CS as a continuous wave optical source. The system transports full-duplex 10 Gbit/s pseudo random sequence that is Orthogonal Frequency Division Multiplexing (OFDM) modulated with 4QAM (Quadrature Amplitude Modulation) mapping over $60 \mathrm{GHz}$ RF carrier that is modulated on optical carrier. The system performance is shown in Fig. 6 for full-duplex RoF signal transportation over $1.5 \mathrm{Km}$ of MMF length and the performance severely depends on the fiber attenuation, dispersion impairment.

\section{CONCLUSION}

In this paper, the convergence of wireless and optical communication technology for deploying and providing the future super broadband services has been presented. Fiber optic transmission has been become one of the most exciting and rapidly dominate infrastructure medium for transportation of the future huge fixed and mobile video on internet by replacing cost effective and ultra fast photonic switching and replacing the active and passive electrical components and devices with the optical equivalent ones. Radio-over-Fiber as a solution has been addressing how to distribute broadband wireless signals in access networks, including dynamic allocation of resources, with deploying the simple and small BSs with centralized operation is possible. The requirement for more bandwidth allocation, places heavy burden on the current operating radio frequency (RF) spectrum and causes spectral congestion at lower microwave frequency. Millimeter wave (MM-Wave) communication system offers a unique way to resolve the bandwidth problems.

\section{REFERENCES}

[1] R. Yuen, X. N. Fernando and S. Krishnan, "Radio Over Multimode Fiber for Wireless Access”, IEEE Canadian Conference on Electrical and Computer Engineering, Vol.3, pp. 1715-1718, May 2004.

[2] The World in 2009, ICT Facts and Figures, "http://www.itu.int”.

[3] Cisco Visual Networking Index: Global Mobile Data Traffic Forecast Update, Jan 2009.
[4] J. Mcdonough, "Moving standards to 100 Gbps and beyond," IEEE Communication Magazine, vol. 45, no.11, pp.6-9, Nov. 2007.

[5] G.-K. Chang, A. Chowdhury, J. Yu, Z. Jia, R. Younce, "Next generation 100Gbit/s Ethernet Technologies,” APOC 2007, Invited Paper, Wuhan China, November 2007.

[6] Miguel de Vega Rodrigo, Guy Latouche, Marie-Ange Remiche, "Modeling bufferless packet-switching networks with packet dependencies” Computer Networks 53 (2009) 1450-1466, February 2009.

[7] N. Larkin, “ASON \& GMPLS; The Battle for the Optical Control Plane";

http://www.dataconnection.com/network/download/whitepapers/ason gmpls.pdf.

[8] E. Le Rouzic and S. Gosselin, "160 Gb/s optical networking : A prospective techno-economic analysis," Journal of Lightwave Technology, Vol 23, No. 10, pp 3024-3033, Oct 2005.

[9] Carla Raffaelli, Kyriakos Vlachos, Nicola Andriolli, Dimitrious Apostolopolus, " Photonic in Switching: Architectures, Systems and enablingtechnologies” Computer Networks Volume 52, Issue 10, Pages 1873-189016, July 2008.

[10] H-C Ji, H. Kim and Y. C. Chung, “ Full-Duplex Radio-Over-Fiber System Using Phase-Modulated Downlink and Intensity-Modulated Uplink”, IEEE Photonics Technology Letters, Vol. 21, No 1, pp 9-11, Jan. 2009.

[11] J. E. Mitchell, "Performance of OFDM at 5.8 GHz using radio over fiber link”, IEE Electronic Letters, Vol. 40, No. 21, pp, Oct. 2004.

[12] S. Chahine, U. A. K Okonkwo and R. Ngah, "Study the Performance of OFDM Radio over Fiber for Wireless Communication Systems”, IEEE INTERNATIONAL RF AND MICROWAVE CONFERENCE PROCEEDINGS, PP 335-338, 2008.

[13] Delivering the optimal upgrade path for 3G networks,http://www.nokia.com/NOKIA_COM_1/Press/Press_Event S.

[14] Rysavy Research, "Edge, HSPA and LTE the Mobile Broadband Advantage”, 3G Americas, Sep., 2007.

[15] S. R. Abdollahi, H.S. Al-Raweshidy, S. Mehdi Fakhraie, R. Nilavalan and M. Kamarei, "Full Duplex $60 \mathrm{GHz}$ Millimeter Wave Transmission over Multi-Mode Fiber”, Presented at IEEE Intenational Conference on Ubiquitous and Future Networks, 2010, Korea. 